\title{
BMJ Open Development of a core outcome set for myocardial infarction in clinical trials of traditional Chinese medicine: a study protocol
}

\author{
Ruijin Qiu (D) , ${ }^{1}$ Changming Zhong, ${ }^{1}$ Songjie Han, ${ }^{1}$ Tianmai He, ${ }^{1}$ Ya Huang, ${ }^{1}$ \\ Manke Guan, ${ }^{1}$ Jiayuan Hu, ${ }^{1}$ Min Li, ${ }^{1}$ Yiyi Lin, ${ }^{1}$ Jing Chen, ${ }^{2}$ Hongcai Shang ${ }^{1}$
}

To cite: Qiu R, Zhong C, Han S, et al. Development of a core outcome set for myocardial infarction in clinical trials of traditional Chinese medicine: a study protocol. BMJ Open 2019;9:e032256. doi:10.1136/ bmjopen-2019-032256

- Prepublication history and additional material for this paper are available online. To view these files, please visit the journal online (http://dx.doi. org/10.1136/bmjopen-2019032256).

Received 10 June 2019 Revised 03 October 2019 Accepted 07 November 2019

A) Check for updates

(c) Author(s) (or their employer(s)) 2019. Re-use permitted under CC BY-NC. No commercial re-use. See rights and permissions. Published by BMJ.

${ }^{1}$ Key Laboratory of Chinese Internal Medicine of Ministry of Education and Beijing, Dongzhimen Hospital, Beijing University of Chinese Medicine, Beijing, China

${ }^{2}$ Tianjin University of Traditional Chinese Medicine, Tianjin, China

Correspondence to

Hongcai Shang;

shanghongcai@foxmail.com

\section{ABSTRACT}

Introduction Myocardial infarction (MI) is the most dangerous complication in patients with coronary heart disease. In China, there is an increasing number of randomised controlled trials (RCTs) of traditional Chinese medicine (TCM) for treating MI. However, the inconsistency of outcome reporting means that a large number of clinical trials cannot be included in systematic reviews to provide the best evidence for clinical practice. The aim of this study is to develop a core outcome set (COS) for future TCM clinical trials of MI, which may improve the consistency of outcome reporting and facilitate the synthesis of data across studies in systematic reviews. Methods and analysis We will conduct a systematic review of Ml clinical trials with any intervention. Semistructured interviews will be conducted to obtain the perspectives of patients with Ml. The outcomes from the systematic review and semistructured interviews will be grouped and used to develop a questionnaire. The questionnaire will be developed as a supplement for the TCM syndromes of Ml and will be constructed from the results of a systematic review, existing medical records and a cross-sectional study. Then two rounds of the Delphi survey will be conducted with different stakeholders (TCM experts and Western medicine experts in cardiovascular disease, methodologists, magazine editors and patients) to determine the importance of the outcomes. Only the TCM experts will need to response to the questionnaire for core TCM syndromes. A face-to-face consensus meeting will be conducted to create a final COS and recommend measurement time for each outcome.

Ethics and dissemination This project has been approved by the Ethics Committee of Dongzhimen Hospital, Beijing University of Chinese Medicine. The final COS will be published and freely available.

Trial registration number This study is registered with the Core Outcome Measures in Effectiveness Trials database as study 1243 (available at: http://www.cometinitiative.org/studies/details/1243).

\section{INTRODUCTION}

Myocardial infarction (MI) refers to myocardial injury, necrosis and cardiac troponin increase caused by myocardial ischaemia (an imbalance of oxygen supply). According
Strengths and limitations of this study

- Systematic reviews for clinical trials of myocardial infarction and traditional Chinese medicine (TCM) syndromes will be conducted.

- A core TCM syndrome set will be developed to supplement the core outcome set (COS).

- The Delphi surveys and consensus meeting will involve both patients and health professionals.

- A measurement time will be recommended for the final COS.

Patients will be recruited at a single centre.

to the 'Global Definition of MI' (2018), MI has five subtypes: ST-segment elevation MI (STEMI) and non-ST-segment elevation MI; ischaemia without atherosclerotic plaque erosion, rupture or destruction; typical MI, such as a typical ST-segment elevation ECG, but no troponin blood tests, such as cardiogenic sudden death; MI during percutaneous coronary intervention and MI during surgical coronary artery bypass grafting. ${ }^{1}$ STEMI is the most dangerous complication in patients with coronary heart disease, resulting in an approximately $5 \%-8 \%$ mortality rate in hospitals. ${ }^{2}$ It is a challenge for researchers and clinicians to improve the diagnosis and treatment of MI and improve the prognosis of patients.

In China, some researchers have carried out a number of randomised controlled trials (RCTs) of traditional Chinese medicine (TCM) for treating MI, which are based on therapeutic principles of TCM, such as syndrome differentiation. Syndrome differentiation is the basis of developing therapeutic principles in TCM, which includes the cause, nature and location of pathological changes at a certain stage of the disease. ${ }^{3}$ It can be comprehensively analysed by clinical symptoms and signs through four methods of 
diagnosis: inspection, auscultation and olfaction, interrogation and palpation.

However, there are some problems in these clinical trials; for example, a lack of reporting primary or longterm outcomes, the heterogeneous outcome in different RCTs, ${ }^{45}$ means that some studies cannot be included in a systematic review/meta-analysis to provide a higher level of evidence for clinical practice, which reduces the value of research and causes waste to a certain extent. The outcomes reported in clinical trials have not been independently validated as important outcomes to other stakeholders, such as patients.

\section{Rationale for the development of a COS}

There is no defined core outcome set (COS) for MI. A COS defines the minimum outcomes that should be measured and reported in clinical trials in a specific area of healthcare. ${ }^{6}$ In 2013, the conception of a COS was introduced in the area of TCM. ${ }^{7}$ Although there are more than 10 COSs of TCM (COS-TCM) registered in the Core Outcome Measures in Effectiveness Trials (COMET) database, no results of COS-TCM have been reported.

Syndrome differentiation is the basis of developing therapeutic principles in TCM. In 2017, the Consolidated Standards of Reporting Trials (CONSORT) Extension for Chinese Herbal Medicine Formulas recommended that outcome measures with TCM syndrome should be reported in detail. ${ }^{8}$ In this study, we will use mixedmethods research to develop a COS that includes TCM core syndrome for MI to determine what outcomes to measure and when to measure them. The abovementioned methods have been used in the development of a COS for non-valvular atrial fibrillation in clinical trials of TCM. ${ }^{9}$

We will conduct a systematic review and qualitative interviews to develop a long list of outcomes for MI. Then, we will develop a list of TCM syndromes of MI based on the systematic review, existing medical records and a cross-sectional study. We will carry out two rounds of the Delphi survey with different stakeholders to determine the importance and priority of outcomes and TCM syndromes that should be reported in clinical trials. A consensus meeting will be held to decide the final COS. The COS will be published and used for future clinical trials to improve outcome reporting in MI. Since TCM is mainly practised in China, this research will achieve national consensus in China.

\section{Scope of the COS-TCM}

The objective of this study is to identify the exact outcomes that concern patients with MI among different stakeholders in RCTs of TCM. We intend to consider when and how these outcomes should be measured after the COS is completed. We will use transparent methodology and address 13 minimum Core Outcome Set Standards for Protocol Items (the COS-STAP Statement) in the protocol. ${ }^{10}$

The scope of the COS-TCM is as follows:
1. Health condition: MI.

2. Population: all patients with MI.

3. Interventions: therapies based on the therapeutic principles of TCM, including traditional herbal medicine, acupuncture, cupping, Qigong and other non-drug therapies.

4. Context of use: RCTs.

\section{Registration}

The study is registered on the COMET database as study 1243 (available at: http://www.comet-initiative.org/ studies/details/1243).

\section{METHODS AND ANALYSIS \\ Steering Committee}

We will form a national Steering Committee to support the development of this research. The Steering Committee will include five experts, such as two TCM experts, a Western medicine expert, a methodologist and a journal editor. They will review and confirm the research protocol, make decisions when there is confusion and attend the consensus meeting to facilitate the development of the COS.

\section{Patient and public involvement}

We will recruit patients to participate in semistructured interviews, a cross-sectional study and two rounds of the Delphi survey.

\section{Design}

This COS will be developed in four phases.

In phases 1, we will develop a long list of general outcomes, of which the information sources include systematic reviews and semistructured interviews.

In phases 2, we will develop a list of TCM syndromes; the information sources will include a systematic review, a retrospective study of medical records and a crosssectional survey.

In phases 3, we will carry out a Delphi survey with different stakeholders, such as health professionals and patients. The health professionals will include Chinese medicine experts in cardiovascular disease (clinicians and researchers), Western medicine experts in cardiovascular disease (clinicians and researchers), methodologists in the field of evidence-based medicine, journal editors and so on.

In phases 4 , we will conduct a consensus meeting to determine what outcomes should be measured in the COS and when to measure the outcomes.

A flowchart of the research is shown in figure 1.

\section{Phases 1: Developing a long list of general outcomes}

There are two steps to develop a long list of general outcomes, namely: a systematic review and semistructured interviews, so that the perspectives of different stakeholders can be considered. 


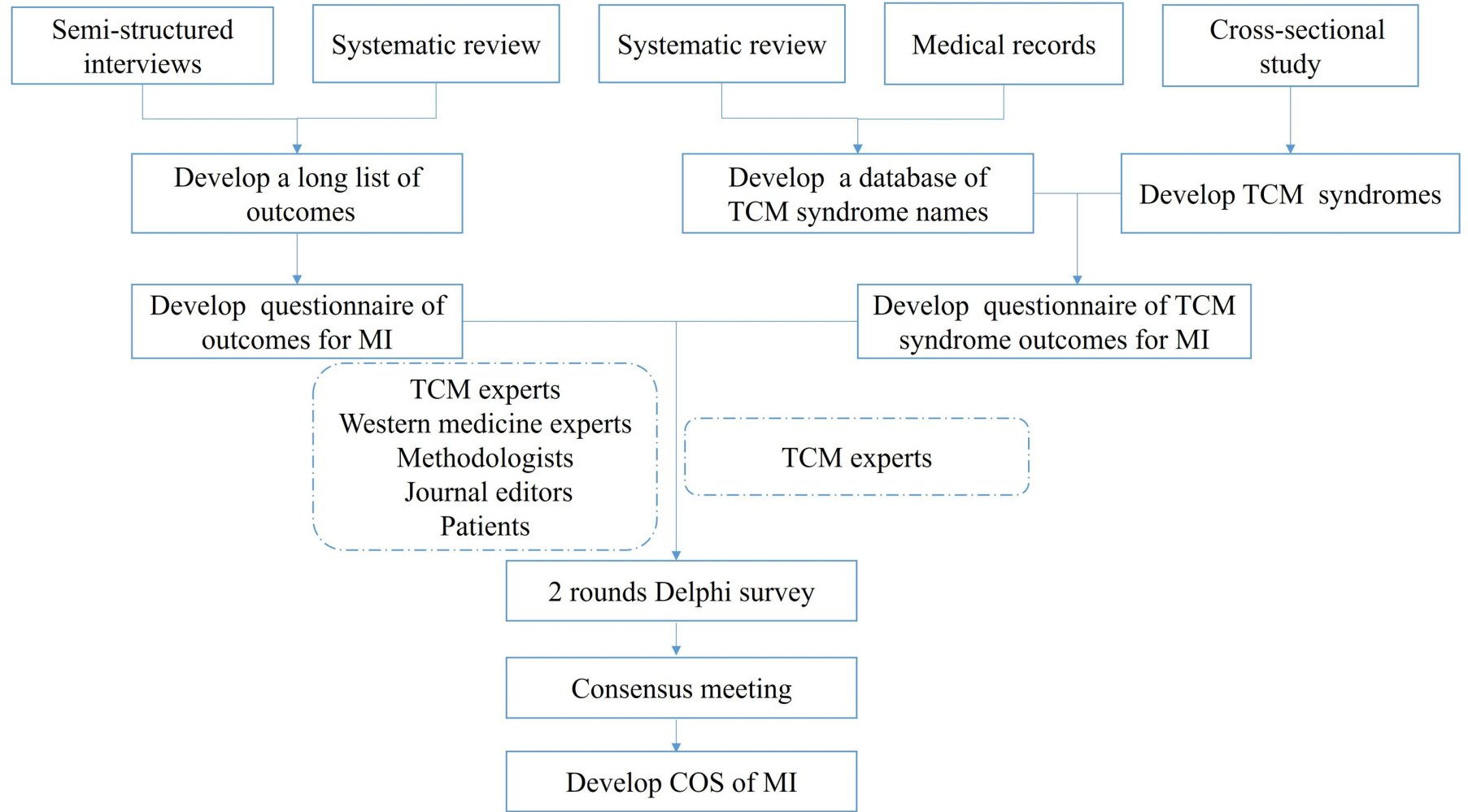

Figure 1 Flowchart for developing a COS for MI in clinical trials of TCM. COS, core outcome set; MI, myocardial infarction; TCM, traditional Chinese medicine.

\section{Step 1: Systematic review}

\section{Search strategy}

We will search three English databases and three Chinese databases: PubMed, the Cochrane Library, Web of Science, Wanfang Database, the China National Knowledge Infrastructure (CNKI) and SinoMed. We will also search the China Clinical Trial Registration Center. The retrieval time range is from 1 January 2009 to 1 January 2019. The search strategy of English databases for general outcome is shown in online supplementary file 1 .

\section{Inclusion criteria and exclusion criteria}

The inclusion and exclusion criteria for the literature are shown in table 1 .

\section{Data extraction}

The data extraction will include the first author's name, number of participants, outcomes (including primary and secondary outcomes), definition of outcomes/ outcome measurement instruments and outcome measurement time (intervention time/follow-up time). If TCM syndromes are reported, TCM syndrome names and diagnostic criteria will also be extracted. In addition, the quality of outcome reporting and the methodological quality will be assessed. There is no standard method to assess the quality of outcome reporting; therefore, we will use the method that has been used by previous research, ${ }^{11} 12$ which includes six items:

1 . Is the primary outcome clearly stated?

2. Is the primary outcome clearly defined so that another researcher would be able to reproduce its measure- ment? Where appropriate, this should include a clear description of time points, the person measuring the outcome, how the outcome was measured (for example, the tools and methods used) and where the outcome was measured.

3. Are the secondary outcomes clearly stated?

4. Are the secondary outcomes clearly defined?

5. Do the authors explain the use of the outcomes they have selected?

6. Are methods used to enhance the quality of outcome measurement (for example, repeated measurement, training) if appropriate?

Table 1 The inclusion and exclusion criteria of systematic review for reported outcomes

\begin{tabular}{|c|c|}
\hline Inclusion criteria & Exclusion criteria \\
\hline $\begin{array}{l}\text { Adult patients with } \\
\text { myocardial infarction }\end{array}$ & $\begin{array}{l}\text { The main objectives of the study } \\
\text { were to assess the mechanisms or } \\
\text { pharmacokinetics of interventions }\end{array}$ \\
\hline $\begin{array}{l}\text { Any kind of interventions } \\
\text { and comparisons is } \\
\text { eligible }\end{array}$ & $\begin{array}{l}\text { Outcome measurement time or } \\
\text { outcome definition/measurement } \\
\text { instruments cannot be extracted }\end{array}$ \\
\hline $\begin{array}{l}\text { Randomised controlled } \\
\text { trials }\end{array}$ & Full-text cannot be obtained \\
\hline $\begin{array}{l}\text { Sample size } \geq 30 \text { in each } \\
\text { group }\end{array}$ & $\begin{array}{l}\text { No information on ethical } \\
\text { approval/funding/trial registration }\end{array}$ \\
\hline $\begin{array}{l}\text { The clinical trials were } \\
\text { published in Chinese or } \\
\text { English }\end{array}$ & \\
\hline
\end{tabular}


Table 2 Inclusion and exclusion criteria for semistructured interviews

\begin{tabular}{ll}
\hline Inclusion criteria & Exclusion criteria \\
\hline Adult patients & $\begin{array}{l}\text { Patients with severe mental } \\
\text { disease }\end{array}$
\end{tabular}

Patients with MI who are

not in acute status

Patients who signed the

informed consent forms

If the eligible study completely meets the item, it will receive 1 point. If it does not meet the item, it will receive 0 points. For objective outcomes, the definition is unnecessary to provide.

We will assess the methodological quality with the Cochrane handbook. ${ }^{13}$ Two researchers will independently extract the information and assess the quality of outcome reporting and the methodological quality of each study. Any disagreement will be resolved by discussion or via consulting with the third investigator.

\section{Step 2: Semistructured interview}

We will recruit patients with MI to participate in semistructured interviews.

\section{Participant selection}

The inclusion criteria and exclusion criteria for semistructured interview are in table 2.

\section{Sampling strategy}

The purpose of semistructured interview is to achieve saturation. According to the experience of previous research, 30 subjects are sufficient to achieve saturation. ${ }^{14}$ However, if there is a new point of view in the final interview, the sample size will be increased. We will recruit patients by purposive sampling. The characteristics of the patients are shown in table 3 .

\section{Recruitment and data collection}

We will approach potential participants at the inpatient ward or outpatient department of Dongzhimen Hospital, Beijing University of Chinese Medicine. An investigator who is trained in qualitative research methods will explain the study to the patients. Then, the patients will receive separate written information sheets to read. The patients who agree to participate in the interview will sign an informed consent form. After that, a semistructured interview will be conducted in the clinical research subject reception room of the hospital. We will collect patients' socioeconomic and demographic information, as well as disease classification during the interview.

The outline of the semistructured interviews are as follows:

1. When were you diagnosed with MI?

2. What inconveniences have you experienced after being diagnosed with MI?

3. What therapies have you received because of MI?

4. What effect do you hope to achieve after treatment?

5. What inconveniences have you experienced from the current treatment?

6. What is the most important outcome for you?

\section{Data analysis}

We will analyse the results of the semistructured interviews concurrently with data collection. All of the interviews will be transcribed verbatim. Then transcriptions will be imported into qualitative analysis software. The data will be analysed by framework methodology, including familiarisation, developing a thematic framework, indexing, devising thematic charts, mapping and interpreting. ${ }^{15}$ Then, narrative explanations of the effects of MI and treatments on the patients' lives will be interpreted by the process of constant comparison to identify outcomes that are important to patients. ${ }^{16}$

\section{Merging outcomes and grouping under outcome domains}

After the systematic review and semistructured interviews are completed, two researchers will merge the outcomes and independently group them under outcome domains. The methods are as follows:

1. If the outcome is in English, it will be translated into Chinese according to the terminology formulated by the National Science and Technology Terminology Committee. If there is no relevant term, it will be determined by two researchers.

2. Composite outcomes will be extracted as individual outcomes.

3. The overlapping outcomes will be merged into one according to the definition of the outcomes. For example, death, death from any cause, mortality, over-

Table 3 Purposive sampling matrix of patients for semistructured interviews

\begin{tabular}{|c|c|c|c|c|c|c|c|c|}
\hline \multirow{2}{*}{$\begin{array}{l}\text { Age } \\
\text { (years) }\end{array}$} & \multirow{2}{*}{$\begin{array}{l}\text { Sample } \\
\text { size }\end{array}$} & \multicolumn{2}{|l|}{ Sex } & \multicolumn{2}{|c|}{ Type of condition } & \multicolumn{3}{|c|}{ Treatment history } \\
\hline & & Male & Female & STEMI & NSTEMI & $\mathrm{PCI}$ & CABG & Thrombolytic \\
\hline $18 \sim 40$ & 6 & 3 & 3 & 3 & 3 & 2 & 2 & 2 \\
\hline $60 \sim 80$ & 9 & 5 & 4 & 5 & 4 & 3 & 3 & 3 \\
\hline$\geq 80$ & 6 & 3 & 3 & 3 & 3 & 2 & 2 & 2 \\
\hline
\end{tabular}

CABG, coronary artery bypass grafting; NSTEMI, ST-segment elevation myocardial infarction; PCl, percutaneous coronary intervention;

STEMI, ST-segment elevation myocardial infarction. 
all mortality, total mortality, all causes of death and all causes of mortality will be aggregated as 'all-cause mortality'.

4. The outcomes that are difficult to merge because there is no definition or measurement instrument to report it will be dropped.

5 . The outcomes will be grouped into different outcome domains according to the taxonomy that has been developed by the COMET initiative. ${ }^{17}$

Then, two researchers will cross-check the results. Any inconsistency will be discussed by the two researchers and to achieve consensus.

\section{Phases 2: Developing a list of TCM syndromes}

In our previous review, we found that TCM syndrome names are nonstandard, so that it is difficult to assess the efficacy of TCM syndromes. ${ }^{18}$ Therefore, we proposed a method to solve the problem in the development of $\mathrm{COS},{ }^{19}$ and it has been used in our previous research. ${ }^{9}$

There are two steps to develop a list of TCM syndromes:

1. Developing a dataset of TCM syndrome names from systematic review and medical records.

2. Investigating TCM syndromes of MI from crosssectional survey.

\section{Step 1: Systematic review}

\section{Search strategy}

We will search three Chinese databases, CNKI, Wanfang Database and SinoMed, to obtain the TCM syndrome names of MI from the researchers' perspective. The retrieval time range is from 1 January 2009 to 1 January 2019. The search terms are in Chinese, with sufficient terminology. The inclusion and exclusion criteria are shown in table 4.

Table 4 The inclusion and exclusion criteria of systematic review for TCM syndrome names

\begin{tabular}{ll}
\hline Inclusion criteria & Exclusion criteria \\
\hline Adult patients & $\begin{array}{l}\text { TCM syndrome names } \\
\text { cannot extracted }\end{array}$ \\
$\begin{array}{l}\text { Any kind of interventions and } \\
\text { comparisons are eligible }\end{array}$ & $\begin{array}{l}\text { The diagnostic criteria for } \\
\text { TCM syndromes cannot } \\
\text { be extracted }\end{array}$ \\
$\begin{array}{l}\text { Both RCTs and observational } \\
\text { studies (case series, cohort } \\
\text { study) are eligible }\end{array}$ & $\begin{array}{l}\text { Full text cannot be } \\
\text { obtained }\end{array}$ \\
\hline
\end{tabular}

Sample size $\geq 30$ in each group

for RCTs, sample size $\geq 50$ in

observational studies

The language is limited to

Chinese, but there is no

restriction for publication status

$\mathrm{RCT}$, randomised controlled trial; TCM, traditional Chinese medicine.
Data extraction

Data extraction will include the first author's name, type of clinical trials, number of participants, TCM syndrome names and syndrome diagnostic criteria, syndrome evaluation items (such as symptoms, tongue coating and pulse) and syndrome measurement methods. Two researchers will independently extract the information, cross-checking will be conducted after completion, and any disagreement will be solved by discussion.

In addition, the names of the syndromes for MI will be obtained from the 'Guidelines for Clinical Research of New Drugs in Traditional Chinese Medicine', 'Traditional Chinese Internal Medicine' and 'Industry Standards of TCM of the People's Republic of China, Diagnostic and Efficacy Standards for TCM Diseases'.

Step 2: Traditional Chinese medicine syndrome names will be identified from medical records

We will conduct a retrospective study of medical records in the last 5years at Dongzhimen Hospital, Beijing University of Chinese Medicine. The aim of this study is to obtain TCM syndrome names for MI from the clinician's perspective. The inclusion and exclusion criteria are as follows:

\section{Inclusion criteria}

1. The first diagnosis was MI.

2. Patients $\geq 18$ years old.

\section{Exclusion criteria}

Patients with severe mental disease.

\section{Data extraction}

Data extraction will include patients' age, sex and TCM syndrome name (if there are more than two TCM syndromes, the first one will be extracted).

\section{Data analysis}

After extracting TCM syndrome names from the systematic review and medical records, a dataset of TCM syndrome names will be developed. Two researchers will extract each syndrome's 'location of disease' and 'feature of disease' independently. Then, they will cross-check and achieve consensus.

Step 3: Cross-sectional survey to investigate TCM syndromes of Ml To obtain the distribution of TCM syndromes for MI, we will conduct epidemiological investigation, such as crosssectional study, to investigate the patients' symptoms and signs.

\section{Sampling size}

We will use convenience sampling to recruit 120 patients with MI in Dongzhimen Hospital, Beijing University of Chinese Medicine.

\section{Participant selection}

The methods of selecting participants are the same as those for semistructured interview. The inclusion criteria 
Table 5 Inclusion criteria and exclusion criteria for crosssectional survey

\begin{tabular}{ll}
\hline Inclusion criteria & Exclusion criteria \\
\hline Adult patients & $\begin{array}{l}\text { Patients with severe mental } \\
\text { disease }\end{array}$
\end{tabular}

Patients with MI who are not

in acute status

\section{Patients who sign the}

informed consent forms

MI, myocardial infarction.

and exclusion criteria for cross-sectional survey are in table 5 .

\section{Recruitment and data collection}

We will approach potential participants at the inpatient ward or outpatient department of. Dongzhimen Hospital, Beijing University of Chinese Medicine. Two Ph.D. students who majored in TCM will collect patients' symptoms and signs through four methods of diagnosisinspection, auscultation and olfaction, interrogation and palpation. They will explain the study and give separate written information sheets to patients. The patients who agree to participate in the study will sign an informed consent form.

To collect data on the symptoms and signs of patients, an information sheet for the four methods of diagnosis will be developed. We will develop the sheet of information with the method that has been used in our previous research. ${ }^{9}$

\section{Data analysis}

From the cross-sectional study, we will obtain and calculate the frequency of symptoms and signs of MI. If the frequency of a symptom or sign is $<5 \%$, it will be removed. Then, we will conduct a clustering analysis for the last data. Two researchers will discuss the classification of TCM syndromes according to the results of the clustering analysis, and then they will extract each syndrome's 'location of disease' and 'feature of disease' independently. Any disagreement will be discussed or the third investigator will be consulted.

Then, the results of 'location of disease' and 'feature of disease' from the cross-sectional survey will be compared with the dataset of TCM syndrome names. If the 'location of disease' and 'feature of disease' extracted from the cross-sectional survey are the same or similar to the 'location of disease' and 'feature of disease' extracted from the dataset of TCM syndrome names, then the TCM syndrome names from the dataset will be used as candidate names.

\section{Phases 3: Delphi survey}

\section{Stakeholder selection}

We will invite health professionals (TCM experts in cardiovascular disease, Western medicine experts in cardiovascular disease, methodologists in the field of evidence-based medicine and journal editors) and patients to participate in two rounds of Delphi survey.

The inclusion and exclusion criteria for health professionals in Delphi survey are as follows:

Inclusion criteria:

1. Health professionals with a bachelor's degree or above.

2. Health professionals who have more than 1 year of work experience.

3. Clinicians who have work experience in tertiary hospitals.

4. There will be no restriction on the professionals' geographical area.

5. The researchers (either first author, corresponding author or other coauthors) have published at least one article regarding clinical trials for cardiovascular disease.

Exclusion criteria: None.

The original information of the health professionals (including TCM experts, Western medicine experts, methodologists and a few journal editors) will be obtained from the membership lists of the Clinical Research Method of Cardiovascular Disease of Professional Committee of Chinese Association of Integrative Medicine and the China Research Institute of China Information Association for Traditional Chinese Medicine and Pharmacy, published papers and the web of journals.

The inclusion and exclusion criteria for patients who will participate in the Delphi survey are as follows:

Inclusion criteria:

1. Patients with MI who do not have acute status.

2. Patients $\geq 18$ years old.

3. There will be no restriction on gender.

4. Patients who have the ability to read and write.

5. Patients who have signed the informed consent forms. Exclusion criteria: patients with severe mental disease.

\section{Sampling strategy}

There is currently no standard sample size calculation method in the Delphi survey. In the previous COS study, the number of professional technicians involved in Delphi study ranged from 12 to 174 technicians. ${ }^{20}$ In this study, we will select 30 stakeholders for each stakeholder group at the beginning. There will be five stakeholder groups, so a total of 150 participants will be recruited. Then, we will use snowball sampling to extend the sample size. Since it is very difficult to understand the TCM terminology for other stakeholders, only TCM experts in cardiovascular disease need to respond to the questionnaire of core TCM syndrome outcomes.

\section{Development of questionnaires for round 1 of the Delphi survey}

We will develop questionnaires for core outcomes and core TCM syndromes of MI separately according to the list of outcomes and TCM syndromes. In round 1 of the Delphi survey, we will collect participants' demographic information. We will use a 9-point scoring system, which has been used in the development of COSs. ${ }^{920}$ Participants 
will have the opportunity to score the importance for all of the outcomes in the questionnaires. A score of ' $1-3$ ' means the outcome is not important to include in the COS, ' $4-6$ ' means the outcome is important but not critical to include in the COS and '7-9' means the outcome is critical to include in the COS. In addition, there is an option of 'unclear' for all candidate outcomes in the questionnaires. At the end of round 1 of the Delphi survey for core outcomes, there will be one open-ended question: which outcomes do you think are important but not included in the questionnaire? The questionnaire will be written in clear language for patients who participate in the Delphi survey, so that it is easier for them to understand.

For the questionnaire on core TCM syndrome outcomes, the symptoms and signs of each TCM syndrome will be presented. The participants will choose a suitable TCM syndrome name according to the characteristics of each TCM syndrome. Then, the participants will have a chance to score all of the symptoms and signs belonging to each classification of TCM syndrome using a 9-point scale. At the end of round 1 of the questionnaire, there will be two open-ended questions for each classification of TCM syndrome:

1. Which TCM syndrome names do you think are appropriate but are not listed in the questionnaire?

2. Which symptoms/signs do you think are important for the TCM syndrome but are not listed in the questionnaire?

\section{Round 1 of the Delphi survey}

Round 1 of the Delphi survey will last for 3 weeks. We will send a personalised email with an outline of the project and questionnaires to the health professionals and invite them to attend the Delphi round 1 within 3 weeks. We will send emails or messages to remind the participants to complete the Delphi survey at the end of the second weekend. To reduce attrition bias, if the response rate is $<80 \%$, we will keep the Delphi rounds open longer.

One of the researchers will approach potential eligible patients at the inpatient ward or outpatient department of Dongzhimen Hospital, Beijing University of Chinese Medicine. The researcher will explain the research to the patients and give separate written information sheets to them. The patients will be asked if they agree to participate in two rounds of the Delphi survey. The ones who agree to participate will sign an informed consent form. Then, the researcher will distribute the printed questionnaire to the patient and ask he/she to complete it. If the patient has any questions, the researcher will answer the questions. The researcher and the patients will make an appointment for the next visit. It will be made clear to the patients that they can withdraw at any time.

\section{Data analysis for round 1 of the Delphi survey}

In this project, we will analyse the results of the health professionals and patients separately. Round 1 of the Delphi survey data analysis will include: the response rate and the frequencies of the response options for each outcome/TCM syndrome name/symptom/sign.

\section{Round 2 of the Delphi survey}

We will summarise each stakeholder's score distribution of each outcome/TCM syndrome name/symptom/sign. In round 2 of the Delphi questionnaire, the participants will receive their score from round 1 of the Delphi questionnaire, and the score distribution of their own stakeholder.

If more than $10 \%$ of participants from any stakeholder group believe that the outcomes/symptoms/signs are important (scored $\geq 4$ ), they will be included in the round 2 of the Delphi survey. In addition, if new outcomes/ TCM syndrome names/symptoms/signs are presented by the participants in round 1 of the Delphi survey, they will be assessed by the research group (the authors) and included in round 2 of the Delphi questionnaire. All of the participants who complete $\geq 90 \%$ of outcomes in round 1 of the Delphi survey will be invited to participate in round 2 of Delphi survey. The participants will have the chance to rescore the importance of outcomes/TCM syndrome names/symptoms/signs.

Round 2 of the Delphi survey will last for 3 weeks. We will send a personalised email outlining the project to stakeholders and invite them to complete round 1 of the Delphi survey within 3 weeks. We will send emails or messages to remind participants to complete the Delphi survey at the end of the second weekend. If the response rate is $<80 \%$, we will keep the Delphi rounds open longer.

\section{Data analysis for round 2 of the Delphi survey}

Round 2 of the Delphi survey data analysis will include the response rate, the frequencies of the response options for each outcome/TCM syndrome name/symptom/ sign, each stakeholder group's score distribution and the attrition bias. We will calculate the average score of each outcome scored by participants who complete or do not complete the two rounds of questionnaires to evaluate if there is attrition bias.

In addition, missing data may result in attrition bias. If there is no attrition bias after calculating the average score of each outcome scored by the participants who complete or do not complete the two rounds of questionnaires, the missing data will not be considered. If there is attrition bias because the participants did not complete round 2 of the Delphi survey, we will calculate the participants' score in round 1 of the Delphi survey. If the missing data are from participants who have not scored all of the outcomes when they return to the questionnaire, then the missing outcome will be considered 'unclear'.

\section{Phases 4: Consensus meeting}

\section{Stakeholder selection}

After completing the Delphi survey, a consensus meeting will be held. We will invite different stakeholders to attend the meeting, regardless of whether the health professionals participate in the Delphi survey. The patients should complete two rounds of the Delphi survey. 
Table 6 The consensus definition

\begin{tabular}{|c|c|c|}
\hline Consensus classification & Description & Definition \\
\hline Consensus in & $\begin{array}{l}\text { The outcome should be included in the } \\
\text { core outcome set }\end{array}$ & $\begin{array}{l}70 \% \text { or more of the participants scored outcome as } 7- \\
9 \text {, and }<15 \% \text { of the participants scored the outcomes } \\
\text { as } 1-3\end{array}$ \\
\hline Consensus out & $\begin{array}{l}\text { The outcome should not be included in } \\
\text { the core outcomes set }\end{array}$ & $\begin{array}{l}50 \% \text { or less of the participants scored the outcome as } \\
7-9\end{array}$ \\
\hline No consensus & $\begin{array}{l}\text { Uncertainty of the importance of } \\
\text { outcome }\end{array}$ & Anything else \\
\hline
\end{tabular}

The inclusion and exclusion criteria for health professionals in the consensus meeting are as follows:

Inclusion criteria:

1. Health professionals with a master's degree or above.

2. Health professionals who have more than 10 years of work experience.

3. Clinicians who have work experience in tertiary hospitals.

4. There will be no restriction on the professionals' geographical area.

Exclusion criteria: None.

\section{Sampling strategy}

There is no standard sample size calculation method for the process of the consensus meeting. To obtain different stakeholders' perspectives, as well as to improve consensus achievement, we will invite 25 participants to attend consensus meeting.

\section{Consensus meeting process}

After completing two rounds of the Delphi survey, a faceto-face consensus meeting will be conducted in Beijing, China. It will last at least 1 day.

The results of round 2 of the Delphi survey will be reported. The number of outcomes that have achieved consensus by all of the stakeholder groups or by any of the stakeholder groups will be reported. The outcomes that have achieved 'consensus in' by all of the stakeholder groups will be the priority for inclusion in the COS. The outcomes that have achieved 'consensus out' by all of the stakeholder groups will be excluded. The remaining outcomes will be discussed by any of the participants in the consensus meeting. Then, the participants will be asked to rescore all of the outcomes that have not achieved consensus by an anonymous method at the meeting. The ones that achieve 'consensus in' will be included in the COS. The ones that achieve 'consensus out' will be excluded from the COS. The remaining outcomes will be discussed by the participants at the consensus meeting. Then, the participants will rescore the remaining outcomes at the meeting. If they still do not achieve consensus on these outcomes, the Steering Committee will decide on the results.

After developing the final COS, the measurement time for the included outcomes will be reported according to the results of the systematic review. The participants will have a chance to discuss them. Then, they will vote on a sufficient measurement time for each outcome with WeChat. The one with the largest proportion will be recommended at the consensus meeting.

\section{Consensus definition}

The consensus definition is given in table 6. We define the consensus according to previous COS research. ${ }^{921}$

\section{ETHICS AND DISSEMINATION}

The entire project has been approved by the Ethics Committee of Dongzhimen Hospital, Beijing University of Chinese Medicine (DZMEC-KY-2019-22). We will obtain informed consent from patients who participate in the semistructured interviews, two rounds of the Delphi survey and cross-sectional study. Informed consent for the other participants is unnecessary.

After the final COS is completed, we will publish this research in a journal, report the results at a conference and disseminate our findings on the website of China Information Association for Traditional Chinese Medicine and Pharmacy Clinical Research Information Association (http://www.cria-cm.net). We will also send the publication to researchers who participated in the Delphi surveys and the consensus meeting so that they can use it in their research.

Acknowledgements We thank the patient advisers for contributing to the research.

Contributors $\mathrm{RQ}, \mathrm{JC}$ and $\mathrm{HS}$ conceived the project, and $\mathrm{RQ}$ and $\mathrm{HS}$ were the principal investigators of the study. CZ, JH and ML are responsible for conducting the systematic review, and $\mathrm{RQ}, \mathrm{SH}, \mathrm{YH}, \mathrm{MG}, \mathrm{YL}$ and $\mathrm{TH}$ are responsible for the management of the clinical research. JC and HS provided supervision for all aspects of the project. RQ wrote the protocol and manuscript and commented on the drafts of this manuscript. All authors have read and approved the manuscript.

Funding This work was supported by the National Key R\&D Program of China (2017YFC1700400, 2017YFC1700402) and the Fundamental Research Funds for the Central Universities (2019-JYB-JS-033).

Competing interests None declared.

Patient consent for publication Not required.

Provenance and peer review Not commissioned; externally peer reviewed.

Open access This is an open access article distributed in accordance with the Creative Commons Attribution Non Commercial (CC BY-NC 4.0) license, which permits others to distribute, remix, adapt, build upon this work non-commercially, and license their derivative works on different terms, provided the original work is properly cited, appropriate credit is given, any changes made indicated, and the use is non-commercial. See: http://creativecommons.org/licenses/by-nc/4.0/.

ORCID iD 
Ruijin Qiu http://orcid.org/0000-0003-3615-4960

\section{REFERENCES}

1 Thygesen K, Alpert JS, Jaffe AS, et al. Fourth universal definition of myocardial infarction (2018). J Am Coll Cardiol 2018;72:2231-64

2 Szummer K, Wallentin L, Lindhagen L, et al. Improved outcomes in patients with ST-elevation myocardial infarction during the last 20 years are related to implementation of evidence-based treatments: experiences from the SWEDEHEART registry 1995-2014. Eur Heart $J$ 2017;38:3056-65.

3 World Health Organization. Who traditional medicine strategy 2014 2023. Geneva, Switzerland: World Health Organization, 2013. http:// apps.who.int/iris/bitstream/10665/92455/1/9789241506090_eng.pdf

4 Li M, Li C, Chen S, et al. Potential effectiveness of Chinese patent medicine Tongxinluo capsule for secondary prevention after acute myocardial infarction: a systematic review and meta-analysis of randomized controlled Trials. Front Pharmacol 2018;9:830.

5 Zhang J, Shang $\mathrm{H}$, Zhang B, et al. Systematic review of randomized controlled trials on treatment of myocardial infarction with YiqiHuoxue Chinese patent medicine. Chin J Tradit Chin Med Title Phar 2008;23:300-6.

6 Williamson PR, Altman DG, Blazeby JM, et al. Developing core outcome sets for clinical trials: issues to consider. Trials 2012;13:132.

7 Zhang L, Zhang J, Chen J, et al. Clinical research of traditional Chinese medicine needs to develop its own system of core outcome sets. Evid Based Complement Alternat Med 2013;2013:1-4.

8 Cheng $\mathrm{C}-\mathrm{W}$, Wu T-X, Shang $\mathrm{H}-\mathrm{C}$, et al. Consort extension for Chinese herbal medicine formulas 2017: recommendations, explanation, and elaboration. Ann Intern Med 2017;167:112-21.

9 Qiu R, Li M, Zhang X, et al. Development of a core outcome set (COS) and selecting outcome measurement instruments (OMIs) for non-valvular atrial fibrillation in traditional Chinese medicine clinica trials: study protocol. Trials 2018;19:541.

10 Kirkham JJ, Gorst S, Altman DG, et al. Core outcome SetSTAndardised protocol items: the COS-STAP statement. Trials 2019;20:116.
11 Durnea CM, Pergialiotis V, Duffy JMN, et al. A systematic review of outcome and outcome-measure reporting in randomised trials evaluating surgical interventions for anterior-compartment vaginal prolapse: a call to action to develop a core outcome set. Int Urogynecol J 2018;29:1727-45.

12 Qiu R, Hu J, Huang Y, et al. Outcome reporting from clinical trials of non-valvular atrial fibrillation treated with traditional Chinese medicine or Western medicine: a systematic review. BMJ Open 2019;9:e028803.

13 The Cochrane Collaboration. Cochrane Handbook for systematic reviews of interventions, 2011. Available: http://handbook-5-1. cochrane.org/ [Accessed 1 Jan 2019].

14 Tugwell P, Boers M. OMERACT conference on outcome measures in rheumatoid arthritis clinical trials: introduction. J Rheumatol 1993;20:528-30.

15 Tugwell P, Boers M, Brooks P, et al. OMERACT: an international initiative to improve outcome measurement in rheumatology. Trials 2007;8:38.

16 Kirkham JJ, Clarke M, Williamson PR. A methodological approach for assessing the uptake of core outcome sets using ClinicalTrials.gov: findings from a review of randomised controlled trials of rheumatoid arthritis. BMJ 2017;357

17 Dodd S, Clarke M, Becker L, et al. A taxonomy has been developed for outcomes in medical research to help improve knowledge discovery. J Clin Epidemiol 2018;96:84-92.

18 Shang HC, XZ W, Qu RJ, et al. Traditional Chinese medicine should not be ignored during the development of precision medicine with Chinese characteristics. TMR 2016;1:105-15.

19 Qiu RJ, Zhang XY, Li M, et al. Significance and method for traditional Chinese medicine syndrome name standardization in the development of core outcome sets. CJTCMP 2018;33:2240-3.

20 Williamson PR, Altman DG, Bagley H, et al. The comet Handbook: version 1.0. Trials 2017;18:280.

21 Fish R, Sanders C, Adams R, et al. A core outcome set for clinical trials of chemoradiotherapy interventions for anal cancer (CORMAC): a patient and health-care professional consensus. Lancet Gastroenterol Hepatol 2018;3:865-73. 